\title{
Implementación de la metodología ABP (Aprendizaje Basado en Problemas) en la enseñanza de los materiales bituminosos para los estudiantes de Arquitectura de Interiores
}

\author{
Sara GuTIÉRrez GONZÁLEZ \\ Universidad de Burgos \\ sggonzalez@ubu.es \\ Ángel RodríGuEz SÁIz \\ Universidad de Burgos \\ arsaizmc@ubu.es
}

\begin{abstract}
Resumen:
Este trabajo describe una experiencia docente de enseñanza-aprendizaje puesta en marcha a través de la metodología del Aprendizaje Basado en Problemas (ABP), llevada a cabo durante tres años con un grupo reducido de alumnos en el módulo de materiales bituminosos de la asignatura de Construcción I de la Titulación de Arquitectura de Interiores de la Universidad de Burgos. Los resultados obtenidos fueron referidos a la idoneidad de las soluciones adoptadas a los encargos realizados, al grado de satisfacción de los alumnos con el método de trabajo y al estudio comparativo de los resultados con las técnicas tradicionales de estudio seguidas hasta ahora.
\end{abstract}

Palabras clave: Aprendizaje Basado en Problemas; Espacio Europeo de Educación Superior; metodología docente

Implementation of PBL methodology (Problem Based Learning) for the bitumen materials in students of Interior Architecture Degree

\begin{abstract}
:
This paper describes the application of the methodology of Problem Based Learning (PBL) in the Course of Construction I of Interior Architecture Degree by University of Burgos. This teaching experience was carried out with a small group of students for three academic years, using bituminous materials module. The results obtained were analyzed taking into account the satisfaction degree of student with the working method. The comparative study of the results obtained was compared with the traditional techniques of study.
\end{abstract}

Key Words: Problem Based Learning; European higher education area; teaching methodology

Referencia normalizada:

Gutiérrez González, S. y Rodríguez Sáiz, A. (2014): Implementación de la metodología ABP (Aprendizaje basado en problemas) en la enseñanza de los materiales bituminosos para los estudiantes de Arquitectura de Interiores. Historia y Comunicación Social. Vol. 19. Núm. Especial Febrero. Págs. 413-425. 
Sumario: 1. Introducción. 2. La metodología del Aprendizaje Basado en Problemas (ABP). 3. Descripción de la experiencia docente. 3.1. Perfil del alumnado. 3.2. Objetivo general. 3.3. Organización de la actividad lectiva. 3.4. Metodología de trabajo. 3.4.1. Tutoría 1. Planteamiento del problema. 3.4.2. Tutoría 2. Conocimiento del material: Estado del Arte. Tormenta de ideas (brainstorming). 3.4.3. Tutoría 3. Análisis de los productos bituminosos. 3.4.4. Desarrollo del encargo profesional. 4. Análisis de los resultados. 5. Conclusiones. 6. Bibliografía.

\section{Introducción}

La adaptación del modelo universitario español al Espacio Europeo de Educación Superior permite la implantación de nuevas metodologías docentes orientadas a la formación en competencias. De acuerdo con este nuevo sesgo metodológico, "la práctica docente debe estar basada en la enseñanza y más en concreto en el aprendizaje del alumno" (Martínez Román, 2009:467), por lo que los docentes deberán reorientar su posición en el proceso de enseñanza-aprendizaje, ya que será "el profesor quien deberá pensar más en el aprendizaje que en la enseñanza, cediendo el protagonismo a los que aprenden y convirtiéndose en auténtico facilitador de los aprendizajes" (Casado, 2006). Esta nueva visión en la forma de enseñar y aprender permite reflexionar sobre la necesidad de formar a los futuros titulados con un nivel de competencias que les permita adaptarse a las necesidades del mercado laboral actual.

El nuevo marco de referencia es más abierto y flexible y permite establecer estrategias docentes de aprendizaje innovadoras y fáciles de comprender por parte del alumnado, pero que requieren una fase previa de experimentación y puesta en práctica, con el objetivo de garantizar el éxito en el proceso de su implantación. En esta primera fase resulta muy útil simular este tipo de estrategias en asignaturas temáticas con contenidos básicos y, posteriormente, aplicarlas a otras más complejas.

La técnica del Aprendizaje Basado en Problemas (ABP) es un procedimiento de trabajo que ha dado buenos resultados cuando se utiliza como metodología de estudio en asignaturas temáticas. Autores como Dochy et al. defienden la validez de aplicación del método ABP en contextos no integrados (2003:533), indicando que: "Los beneficios que se le atribuyen, son el deseo de mejorar la práctica docente e innovar en la enseñanza" (Lobato, 2011:37). En la experiencia que se expone en este artículo, se diseñó un módulo de Aprendizaje Basado en Problemas (ABP) para la asignatura de Construcción I. Esta asignatura se integra en el Programa de Estudios de la Titulación de Arquitectura de Interiores en la Universidad de Burgos y se imparte en el primer curso de la titulación. Este trabajo recoge el estudio realizado durante tres cursos académicos con un grupo reducido de alumnos utilizando el ABP, sirviendo como referencia para establecer hipótesis de comportamiento respecto del desarrollo de capacidades, hábitos de estudio y técnicas de aprendizaje en los alumnos a los que se les ha aplicado el módulo. 


\section{La metodología del aprendizaje basado en problemas (ABP)}

El ABP es una metodología docente que rompe con el esquema tradicional de relaciones alumno-profesor, y que permite que el alumno sea el principal protagonista en el proceso de aprendizaje. De esta forma, la metodología de enseñanza tradicional, en la que el profesor facilita la información al alumno para que éste busque cómo aplicarla en la resolución de un problema, es sustituida por una nueva orientación del proceso, siendo el alumno, mediante la reflexión y la investigación, el que determina cómo resolver el problema planteado por el profesor. Como indica Barrows, el ABP es un "un método de aprendizaje basado en el principio de usar problemas como punto de partida para la adquisición e integración de los nuevos conocimientos" (1986:481). De esta forma, los estudiantes ocupan el "centro activo" del proceso de aprendizaje, asumiendo su protagonismo de forma relevante.

Otros autores, como Prieto, reafirman el enfoque del aprendizaje activo señalando que "el aprendizaje basado en problemas representa una estrategia eficaz y flexible que, a partir de lo que hacen los estudiantes, puede mejorar la calidad de su aprendizaje universitario en aspectos muy diversos" (2006:173). Por todo ello, la técnica del ABP es una metodología que responde a los objetivos del proceso de enseñanza-aprendizaje, ya que contribuye al impulso de las competencias transversales que todo alumno universitario debe adquirir durante su proceso de formación, desarrollando capacidades tales como la toma de decisiones, la integración en el grupo para el trabajo en equipo o la capacidad de comunicar sus experiencias a sus profesores y compañeros.

De igual forma, el ABP es una técnica que estimula la creatividad de alumno, ya que éste asume la responsabilidad de buscar las fuentes de información y gestionar su contenido, desarrollando habilidades investigadoras sobre el problema propuesto por el profesor, para la búsqueda de una solución adecuada al mismo. Como indican Benito y Cruz, el ABP "favorece el desarrollo del razonamiento eficaz y la creatividad"(2005). Por tanto, son muchos los beneficios que reporta el ABP, siendo evidente el "aumento de habilidades en la resolución autónoma de problemas" (Gijbelts, 2005:27).

Exley y Dennick definen algunas de las características más importantes de la metodología ABP, destacando especialmente "el trabajo autónomo y en equipo, participación de un número reducido de alumnos por grupo, posibilidad de interrelacionar distintas materias o disciplinas académicas y la nueva función del profesor, que ocupa el rol de tutor y actúa facilitando el aprendizaje del alumno" (2007).

\section{Descripción de la experiencia docente}

Este trabajo muestra el estudio realizado con alumnos de la Asignatura de Construcción I del Primer Curso de la Titulación de Arquitectura de Interiores de la 
Universidad de Burgos durante los Cursos Académicos 2010-11, 2011-12 y 2012-13. La asignatura se imparte en el primer semestre del curso.

Durante el proceso de implantación del Espacio Europeo de Educación Superior, la Universidad de Burgos ha organizado programas de formación específicos, a través del Instituto de Formación e Innovación Educativa, con el objetivo de facilitar al profesorado una formación suficiente sobre las nuevas metodologías docentes de trabajo, garantizando su implementación en el aula. El ABP toma especial protagonismo al ser una técnica de estudio muy adecuada para las disciplinas de carácter técnico. Es en este contexto en el que se enmarca la experiencia docente que aquí se expone.

El tema elegido para el desarrollo del método fue el de "Materiales Bituminosos", diseñando un problema para que los alumnos conocieran los objetivos a alcanzar en el estudio de esta materia.

\subsection{Perfil del alumnado}

Con el objetivo de conocer el perfil del alumnado matriculado en la asignatura se propone rellenar un cuestionario básico de referencias. De su estudio destacan las siguientes conclusiones:

Los alumnos son estudiantes recién incorporados a la Universidad. Son creativos y muestran interés por el diseño. Destacan por su alto nivel de autonomía en la búsqueda de información a través de Internet. Sin embargo, muestran una escasa utilización de las técnicas de estudio y una gran dependencia del profesor en el proceso de aprendizaje.

En la Tabla 1 se muestra el número de alumnos que han participado en el programa educativo ABP que, como se ha indicado anteriormente, se ha desarrollado durante tres cursos académicos. Como se puede observar, todos los cursos registraron una participación inferior a 10 alumnos, lo que posibilitó realizar esta experiencia docente de manera controlada y personalizada.

Tabla 1. Número de alumnos que han participado en el programa ABP

\begin{tabular}{ccc}
\hline Curso & $\begin{array}{c}\text { Número total de } \\
\text { alumnos }\end{array}$ & $\begin{array}{c}\text { Alumnos } \\
\text { ABP }\end{array}$ \\
\hline $\mathbf{2 0 1 0 - 1 1}$ & 14 & 9 \\
\hline $\mathbf{2 0 1 1 - 1 2}$ & 8 & 8 \\
\hline $\mathbf{2 0 1 2}-13$ & 8 & 8 \\
\hline
\end{tabular}

. Fuente: Elaboración propia 


\subsection{Objetivos general}

La experiencia docente que se muestra en este trabajo tiene como objetivo exponer que la metodología de estudio ABP, aplicada a una unidad temática de la asignatura de Construcción I para los alumnos del primer curso de la Titulación de Arquitectura de Interiores de la Universidad de Burgos, contribuye positivamente al proceso de aprendizaje del alumno. Con este trabajo se analiza el grado de mejora obtenido en el proceso de enseñanza, especialmente referido a la comprensión de la materia objeto de estudio, a la capacidad de comunicación y a la motivación e implicación del estudiante en su propio proceso de aprendizaje.

\subsection{Organización de la actividad lectiva}

La asignatura de Construcción I de la Titulación de Arquitectura de Interiores es de carácter obligatorio y cuenta con 12 créditos ( 9 teóricos y 3 prácticos). La docencia de asignatura se organiza en una sesión semanal de cuatro horas de duración, a lo largo de un curso académico completo. La experiencia docente se realizó durante dos horas semanales, en la mitad del segundo semestre del curso académico, con un total de 6 sesiones de trabajo en aula.

Con respecto a los recursos físicos disponibles para el aprendizaje, se utilizaron dos tipos de aula: el Laboratorio de Materiales de Construcción y el Aula de Informática, dotada ésta con equipos informáticos y acceso a Internet.

\subsection{Metodología de trabajo}

El trabajo se distribuyó en cuatro tutorías dirigidas al colectivo de alumnos, desarrollando en cada una las diferentes etapas en las que se estructura esta metodología de estudio.

La primera tutoría se dedicó a exponer el proceso de aprendizaje y se formaron los grupos de trabajo. Posteriormente, se enunció el problema que recoge el "encargo profesional" sobre el que debe trabajar cada grupo.

Una vez planteado el problema objeto de estudio, en la segunda tutoría se propuso desarrollar una tormenta de ideas (brainstorming), poniendo en común todo el conocimiento previo, con el objetivo de que los alumnos identifiquen lo que saben sobre el material objeto de estudio.

En una tercera etapa se analizaron los productos bituminosos existentes en el mercado de aplicación a los casos propuestos, mediante la realización de ensayos de laboratorio, proyección de vídeos temáticos y debate en clase. Esta tutoría tuvo como objetivo completar la estructura de los conocimientos necesarios que el alumno debe adquirir para realizar su encargo profesional.

Continuando con el proceso metodológico, en una cuarta tutoría se materializa el compromiso de los alumnos con el trabajo en grupo, aceptando el encargo propuesto y gestionando la información adquirida en las tutorías precedentes. El profesor actúa 
como asesor en el proceso y estimula el razonamiento de los alumnos mediante la técnica de la pregunta-respuesta.

Como colofón, se propone la exposición de los trabajos realizados, donde los alumnos explican a sus compañeros las estrategias de trabajo seguidas, las dificultades surgidas, las soluciones propuestas y el resultado final del encargo docente.

El proceso se retroalimenta con una evaluación colectiva de cada uno de los alumnos respecto de sus compañeros, señalando los puntos fuertes, los puntos débiles y las mejoras que, en cada caso, pudieran aplicarse a las situaciones planteadas.

\subsubsection{Tutoría 1. Planteamiento del problema.}

En la primara tutoría el profesor expone el problema y analiza su contenido, con el objetivo de que los alumnos entiendan su enunciado y lo que se les solicita. Posteriormente, el profesor organiza los grupos, formados por no más de tres personas.

En una exposición posterior se expone de manera más pormenorizada el problema propuesto, orientándolo a una situación real de dificultad a la que no podemos dar respuesta, por no disponer de los conocimientos necesarios para resolverla.

En esta primera fase sólo se pretende que los grupos tomen conciencia del problema planteado, sin esperar que lo resuelvan, al no disponer de la base de conocimientos necesaria.

El problema propuesto en este módulo se presentó a los alumnos con el título de “encargo profesional”, desglosándolo en subproblemas para estructurar y guiar el trabajo (Figura 1).

Figura 1. Encargo profesional de ejecución y promoción de uso de productos bituminosos en una sala de fiestas con piscina exterior.

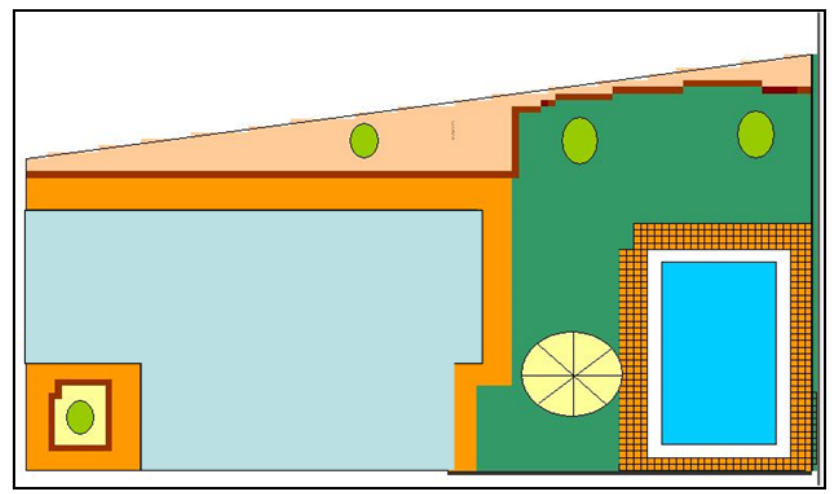

Fuente: Elaboración propia.

A su vez, el problema se dividió en cinco subproblemas, cuyos enunciados y planteamientos proporcionaban al alumno una visión general y estructurada de la 
situación planteada. Los subproblemas, a su vez, se organizaron en actividades, con el objetivo de facilitar a los alumnos las pautas necesarias para acometer el encargo profesional final. Para ello, se establecieron un total de 5 actividades, con el propósito de que los alumnos adquirieran las herramientas necesarias para resolver los subproblemas planteados y, finalmente, afrontar la resolución del "encargo profesional" propuesto. Se trataba de actividades a las que hay que aplicar procesos lógicos de deducción sencillos, en los que el alumno debe poner en práctica su razonamiento personal reflexivo.

3.4.2 Tutoría 2. Conocimiento del material: Estado del Arte. Tormenta de ideas (brainstorming)

En esta segunda sesión se puso en práctica la metodología de resolución de problemas, estableciendo como objetivo conocer qué es un material bituminoso en su sentido más amplio y genérico, diferenciando las materias primas utilizadas en su obtención y distinguiendo los productos auxiliares y elaborados obtenidos a partir de ellos. De nuevo se organizó una tormenta de ideas (brainstorming) para que los alumnos tomaran conciencia de la situación a la que se enfrentaban. Al mismo tiempo, se adquirían los conocimientos necesarios para acometer el resto de actividades. Realizada la lluvia de ideas, éstas se organizaron por categorías (Figura 2).

Figura 2. Tormenta de ideas

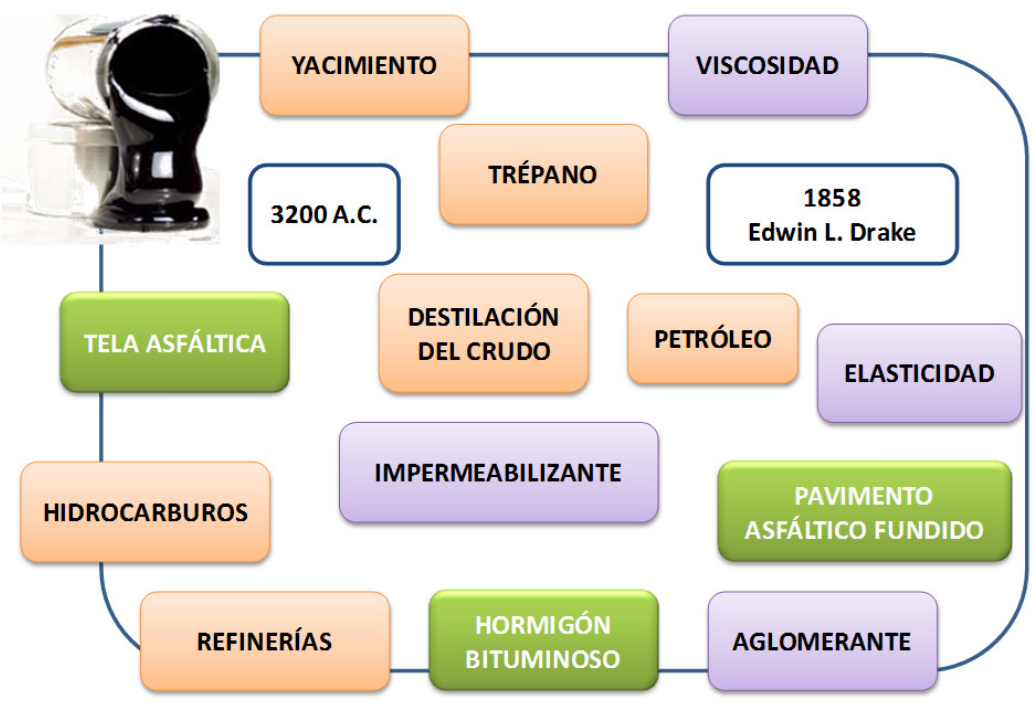

Fuente: Elaboración propia 


\subsubsection{Tutoría 3. Análisis de los productos bituminosos.}

Las actividades de resolución del subproblema (análisis de productos bituminosos) se desarrollaron a través de:

Prácticas de laboratorio. Con esta actividad los alumnos adquieren habilidades propias de los métodos de investigación científica, profundizando y consolidando los fundamentos teóricos referentes a los productos bituminosos, mediante el método experimental. A cada alumno se le facilitó una "ficha de laboratorio", en la que se detallaba el objetivo del ensayo a realizar, los materiales utilizados y los equipos necesarios en la experimentación.

Visionado de videos. En esta sesión se proyectan vídeos divulgativos sobre materiales bituminosos, como complemento a las explicaciones del profesor. Con esta técnica, al mismo tiempo que el alumno descansa, adquiere conocimientos, estimulando su atención sin que la actividad docente se interrumpa.

Ejercicios y debate en aula. Con esta actividad el docente controla el proceso del aprendizaje del alumno y las competencias adquiridas en fases precedentes. El profesor informa a los alumnos del grado de aprovechamiento seguido mediante la publicación de las calificaciones obtenidas, lo que conlleva a una toma en consideración por parte del alumno sobre su nivel formativo, asumiendo la responsabilidad para dirigir su propio aprendizaje.

\subsubsection{Tutoría 4. Desarrollo del encargo profesional.}

La cuarta sesión lectiva se caracterizó por ser la más exigente de las actividades desarrolladas en el módulo ABP. En esta sesión los alumnos deben mostrar el trabajo realizado, su planificación y las iniciativas tomadas al respecto.

Los grupos distribuyeron los trabajos entre sus integrantes, poniendo en práctica el trabajo colaborativo de reparto de tareas entre sus miembros. De esta forma, desarrollaron habilidades para la búsqueda de fuentes de información y el manejo y gestión de los contenidos.

Esta actividad, cuya problemática lleva el nombre de "encargo profesional", se formuló con el siguiente enunciado:

El trabajo de cada equipo consiste en preparar una presentación de información y promoción de la obra que se le ha sido encargada, con una extensión máxima de 10 diapositivas y un tiempo límite de exposición de 10 minutos.

El profesor se limita a transmitir unas nociones básicas, motivando a los alumnos en la búsqueda de la información adicional necesaria para alcanzar y verificar la solución aportada.

Como recursos de apoyo se facilitaron enlaces a páginas Web de fabricantes, con el objetivo de buscar información precisa sobre materiales bituminosos, tanto en impermeabilización como asfaltos para suelos y pavimentación. 
Como se ha indicado, los alumnos integrantes de cada curso se dividieron en cuatro grupos variables, compuestos por 2 y 3 participantes. El desarrollo de esta tarea se realizó en el Aula de Informática durante dos sesiones, a modo de taller de trabajo. La primera sesión se dedicó a preparar y orientar el encargo profesional, resolviendo las dudas surgidas sobre el planteamiento y contenidos del trabajo a desarrollar. En la segunda sesión se realizaron las exposiciones públicas de cada grupo.

Se propuso la retroalimentación colectiva de forma que cada grupo expuso su trabajo al resto de alumnos, siendo éstos los evaluadores de sus compañeros, de forma que "el alumno sea capaz de reflexionar sobre su propio proceso de construcción del conocimiento y, por tanto, sobre su aprendizaje" (Zabit, 2006:19).

Con el objetivo de fomentar el sentido crítico, los alumnos dispusieron de un guión con los criterios a tener en cuenta en la evaluación de los trabajos. El profesor actuó como corrector del proceso, reservándose únicamente el derecho de mejorar la nota final obtenida.

Los alumnos recibieron la información expuesta en los trabajos, reflexionando sobre los contenidos incorrectos, insuficientes y las mejoras que pudieran aportarse.

\section{Análisis de los resultados}

La evaluación final del trabajo realizado se obtuvo mediante la asignación de diferentes puntuaciones a las actividades que integraban cada módulo ABP, aplicando la técnica de la "evaluación continua" (Figura 3)

Figura 3. Pesos asignados a las deferentes actividades que integran el módulo

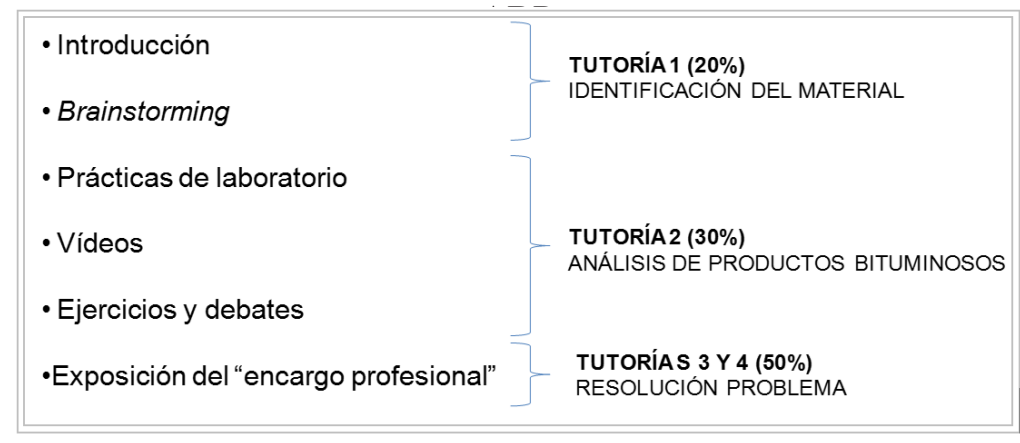

Fuente: Elaboración propia

Los criterios de evaluación se establecieron atendiendo al grado de adecuación del trabajo realizado a los enunciados propuestos, su nivel de desarrollo, la calidad de la investigación, la aportación de información, precisión conceptual etc. 
Para conocer el grado de satisfacción del alumno respecto de la experiencia desarrollada, se diseñó una encuesta basada en 9 preguntas, que fue entregada a los alumnos para su cumplimentación al finalizar el módulo (Figura 4).

Se establecen cinco grados de satisfacción, a los que los alumnos pudieron responder puntuando de 1 a 5 , de acuerdo con las siguientes referencias:

1: Ninguna; 2. Poca; 3: Normal; 4: Buena; 5: Excelente.

Del análisis de las respuestas obtenidas, al 78\% de los alumnos les pareció interesante para su futura profesión el conocimiento de los materiales bituminosos; un $89 \%$ opinó que el contenido del módulo fue sencillo y fácil de comprender; tanto la asistencia a clase como las actividades prácticas han permitido comprender mejor la materia a la totalidad de los alumnos; un $89 \%$ enfatiza la figura del profesor en su función de animador del proceso, motivando a la participación activa del grupo en el desarrollo de las clases. Respecto a los comentarios personales de los alumnos, la mayoría concluyó que, tanto los conocimientos adquiridos como la metodología utilizada contribuyeron a su aprendizaje global y autónomo.

\section{Conclusiones}

El análisis de los resultados permite establecer las siguientes conclusiones:

Los resultados del proceso de aprendizaje obtenidos mediante la aplicación de la metodología $\mathrm{ABP}$ al estudio de un módulo concreto de la asignatura de Construcción I de la Titulación de Arquitectura de Interiores de la Universidad de Burgos han sido muy satisfactorios, registrando un mayor aprovechamiento por parte del alumnado, en comparación con las técnicas de aprendizaje tradicionales centradas en el protagonismo del profesor.

Se comprueba que el ABP incentiva la implicación del alumnado en el proceso de aprendizaje, observando un alto grado de cumplimiento de las actividades propuestas y una empatía con la metodología de trabajo desarrollada.

De igual forma, tanto la evaluación continua del proceso de enseñanza-aprendizaje mediante ABP como las estrategias de retroalimentación del método, permiten comprobar una valoración muy positiva de la calidad de los conocimientos adquiridos por el alumnado.

Tanto el proceso de diseño del método de trabajo ABP como su implantación en el sistema de enseñanza-aprendizaje, así como los procedimientos de validación y seguimiento del mismo, representan un volumen de trabajo extremadamente costoso en tiempo para el profesorado. Los responsables del actual modelo universitario deberían fomentar su implantación y reconocer el esfuerzo y la dedicación de los profesores comprometidos con la innovación. 
Figura 4. Encuesta de satisfacción de los alumnos que participaron en el programa ABP.

Rellena las casillas según tu grado de conformidad:

$\begin{array}{lllll}\text { 1. No 2. Un poco } & \text { 3. Normal } & \text { 4. Bastante 5. Mucho }\end{array}$ materiales bituminosos

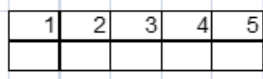

2. El contenido sobre el tema de materiales bituminosos me ha parecido sencillo y fácil de comprender

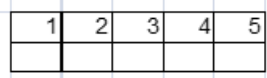

3. La asistencia a clase me ha permitido comprender mejor la materia

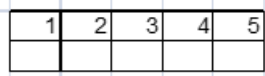

4. Las actividades práctic as son un buen complemento a los contenidos teóricos de la materia

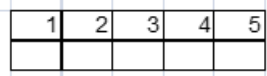

5. El profesor nos motiva a que partic ipemos de forma activa en el desarrollo de la clase, consiguiendo aumentar mi interés por la materia

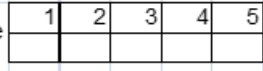

6. El profesor informa con claridad sobre el programa y método de evaluación

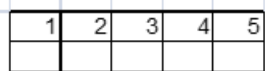

7. El profesor organiza y estructura bien las clases
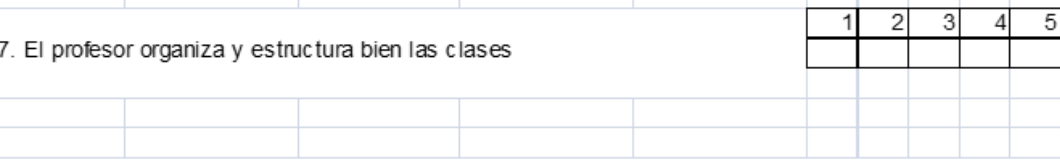

8. Señana al menos un aspecto que te haya parecido positivo sobre la parte de materiales bituminosos

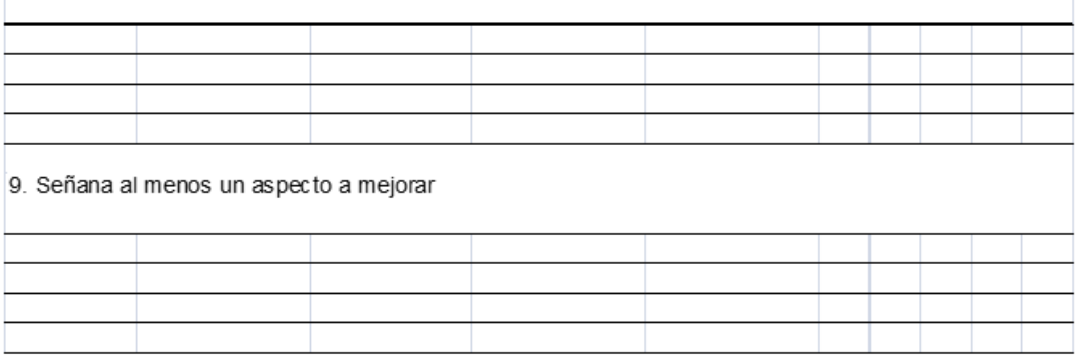

Fuente: Elaboración propia 


\section{Bibliografía}

\subsection{Libros:}

BENITO, A. Y CRUZ, A. (2005). Nuevas claves para docencia universitaria en el Espacio Europeo de Educación Superior. Madrid: Narcea.

EXLEY, K. Y DENNIS, R. (2007). Enseñanza en pequeños grupos en Educación Superior. Madrid: Narcea.

6.2 Capítulos o artículos en libros o revistas en papel:

BARROWS, H.S. (1986). "A Taxonomy of problem-based learning methods, en Medical Education", 20/6, 481-486.

DOCHY, F., MIEN SEGERS, M., PIET Y VAN DER BOSSCHE, P. (2003). “Effects of Problem-based learning: a meta-analysis". Learning and Instruction 13, 533-568.

GIJBELS, D., DOCHY, F.,VAN DEN BOSSCHE,P., SEGERS, M. (2005). „Effects of problem-based learning: A meta-analysis from the angle of assessment". Review of Educational Research, 75 (27).

LOBATO, C. MEDINABEITIA, A. (2011). "Perfiles Motivacionales del Profesorado ante la Formación en Metodologías Activas en la Universidad". Formación Universitaria, 4(1), 37-48 (2011).

MARTÍNEZ ROMÁN, María Asunción (2009): “Aplicación del aprendizaje basado en problemas a la formación en competencias de trabajadores sociales". Propuestas de diseño, desarrollo e innovaciones curriculares y metodología en el EEES. Universidad de Alicante, Alicante, Marfil, Alcoy, España, 467-484.

PRIETO, L. (2006). “Aprendizaje basado en problemas, en Miscelánea Comillas. Revista de Ciencias Humanas y Sociales Vol.64. Núm.124. Págs. 173-196.

ZABIT, M.M (2006). Problem-Based Learning On Students. Critical Thinking Skills In Teaching Business Education In Malaysia: A Literature Review". American Journal of Business Education 3 (6), 19-32.

6.3 Artículos en publicaciones web:

CASADO ORTIZ, Rafael (2006): Convergencia con Europa y cambio en la universidad. Los profesores y las nuevas tecnologías como elementos clave en el nuevo modelo de aprendizaje del Espacio Europeo de Educación Superior, en Revista electrónica de Tecnología Educativa, $\mathrm{n}^{\circ} 20$. Disponible en: http://edutec.rediris.es/ Revelec2/revelec20/casado20.pdf. [19-09-2013]. 


\section{Los autores}

Dra. Sara Gutiérrez-González. Dra. Ingeniero en Organización Industrial y Arquitecto Técnico por la Universidad de Burgos. Directora del Área de Construcciones Arquitectónicas de la Universidad de Burgos.

Cuenta con una amplia experiencia profesional en el sector de la construcción y como docente e investigadora universitaria, ejerciendo actualmente como Profesora Ayudante Doctor adscrita al Departamento de Construcciones Arquitectónicas e I.C.T. en la Escuela Politécnica Superior de la Universidad de Burgos. Pertenece al Grupo de Investigación en Ingeniería de Edificación, donde ha publicado numerosos artículos internacionales, patentes de invención y comunicaciones a congresos, todo ello referido a la línea de investigación del campo de reciclado de materiales. Forma parte del Grupo de Innovación docente de Materiales de Construcción donde ha participado en numerosas publicaciones, ponencias a congresos educativos y artículos de investigación relacionados con el Espacio Europeo de Educación Superior.

Dr. Ángel Rodríguez Sáiz. Dr. Ingeniero de Organización Industrial por la Universidad de Burgos y Arquitecto Técnico y Licenciado en Derecho por la Universidad de Valladolid. Director del Departamento de Construcciones Arquitectónicas e Ingenierías de la Construcción y del Terreno de la Universidad de Burgos.

Profesor Titular de Universidad, ha desarrollado su carrera profesional en diferentes empresas del sector de la construcción. Forma parte del Grupo de Investigación en Ingeniería de Edificación, donde ha participado en la publicación de numerosos artículos, ponencias a congresos y patentes de invención sobre reciclado de materiales y reutilización de subproductos industriales. Participa en actividades de investigación docentes integrado en el Grupo de Innovación Docente de Materiales de Construcción, elaborando material de apoyo a la enseñanza y participando en congresos educativos y revistas docentes especializadas. 